\title{
Strong convergence of an iterative process for a family of strictly pseudocontractive mappings
}

\author{
Yuan Qing ${ }^{1}$, Sun Young Cho ${ }^{2}$ and Meijuan Shang ${ }^{3,4^{*}}$
}

\section{"Correspondence:}

meijuanshang@yahoo.com.cn

${ }^{3}$ Department of Mathematics,

School of Science, Beijing Jiaotong

University, Beijing, 100044, China

${ }^{4}$ Department of Mathematics,

Shijiazhuang University,

Shijiazhuang, 050035, China

Full list of author information is

available at the end of the article

\begin{abstract}
In this article, fixed point problems of a family of strictly pseudocontractive mappings are investigated based on an iterative process. Strong convergence of the iterative process is obtained in a real 2-uniformly Banach space.

MSC: 47H09; 47J05; 47J25
\end{abstract}

Keywords: accretive operator; iterative process; fixed point; nonexpansive mapping; zero point

\section{Introduction and preliminaries}

Throughout this paper, we always assume that $E$ is a real Banach space. Let $E^{*}$ be the dual space of $E$. Let $J_{q}(q>1)$ denote the generalized duality mapping from $E$ into $2^{E^{*}}$ given by

$$
J_{q}(x)=\left\{f^{*} \in E^{*}:\left\langle x, f^{*}\right\rangle=\|x\|^{q},\left\|f^{*}\right\|=\|x\|^{q-1}\right\}, \quad \forall x \in E,
$$

where $\langle\cdot, \cdot\rangle$ denotes the generalized duality pairing. In particular, $J_{2}$ is called the normalized duality mapping, which is usually denoted by $J$. In this paper, we use $j$ to denote the singlevalued normalized duality mapping. It is known that $J_{q}(x)=\|x\|^{q-2} J(x)$ if $x \neq 0$. If $E$ is a Hilbert space, then $J=I$, the identity mapping. Further, we have the following properties of the generalized duality mapping $J_{q}$ :

(1) $J_{q}(t x)=t^{q-1} J_{q}(x)$ for all $x \in E$ and $t \in[0, \infty)$;

(2) $J_{q}(-x)=-J_{q}(x)$ for all $x \in E$.

A Banach space $E$ is said to be smooth if the limit

$$
\lim _{t \rightarrow 0} \frac{\|x+t y\|-\|x\|}{t}
$$

exists for all $x, y \in U_{E}$. It is also said to be uniformly smooth if the limit is attained uniformly for all $x, y \in U_{E}$. The norm of $E$ is said to be Fréchet differentiable if, for any $x \in U_{E}$, the above limit is attained uniformly for all $y \in U_{E}$. The modulus of smoothness of $E$ is the function $\rho_{E}:[0, \infty) \rightarrow[0, \infty)$ defined by

$$
\rho_{E}(\tau)=\sup \left\{\frac{1}{2}(\|x+y\|+\|x-y\|)-1:\|x\| \leq 1,\|y\| \leq \tau\right\}, \quad \forall \tau \geq 0 .
$$

() 2013 Qing et al.; licensee Springer. This is an Open Access article distributed under the terms of the Creative Commons Attribution License (http://creativecommons.org/licenses/by/2.0), which permits unrestricted use, distribution, and reproduction in any medium, provided the original work is properly cited. 
The Banach space $E$ is uniformly smooth if and only if $\lim _{\tau \rightarrow \infty} \frac{\rho_{E}(\tau)}{\tau}=0$. Let $q>1$. The Banach space $E$ is said to be $q$-uniformly smooth if there exists a constant $c>0$ such that $\rho_{E}(\tau) \leq c \tau^{q}$. It is shown in [1] that there is no Banach space which is $q$-uniformly smooth with $q>2$. Hilbert spaces, $L^{p}$ (or $l^{p}$ ) spaces and Sobolev space $W_{m}^{p}$, where $p \geq 2$, are 2uniformly smooth.

Let $C$ be a nonempty closed convex subset of $E$ and let $T: C \rightarrow C$ be a mapping. In this paper, we use $F(T)$ to denote the fixed point set of $T$. A mapping $T$ is said to be $\kappa$ contractive iff there exists a constant $\kappa \in(0,1)$ such that

$$
\|T x-T y\| \leq \kappa\|x-y\|, \quad \forall x, y \in C .
$$

A mapping $T$ is said to be nonexpansive iff

$$
\|T x-T y\| \leq\|x-y\|, \quad \forall x, y \in C .
$$

A mapping $T$ is said to be $\kappa$-strictly pseudocontractive iff there exist a constant $\kappa \in(0,1)$ and $j(x-y) \in J(x-y)$ such that

$$
\langle T x-T y, j(x-y)\rangle \leq\|x-y\|^{2}-\kappa\|(I-T) x-(I-T) y\|^{2}, \quad \forall x, y \in C .
$$

It is clear that (1.1) is equivalent to the following:

$$
\langle(I-T) x-(I-T) y, j(x-y)| \geq \kappa\|(I-T) x-(I-T) y\|^{2}, \quad \forall x, y \in C .
$$

The class of $\kappa$-strictly pseudocontractive mappings was first introduced by Browder and Petryshyn [2] in Hilbert spaces. A mapping $T$ is said to be pseudocontractive iff there exists $j(x-y) \in J(x-y)$ such that

$$
\langle T x-T y, j(x-y)\rangle \leq\|x-y\|^{2}, \quad \forall x, y \in C .
$$

A mapping $T$ is said to be $\kappa$-strongly pseudocontractive iff there exist a constant $\kappa \in(0,1)$ and $j(x-y) \in J(x-y)$ such that

$$
\langle T x-T y, j(x-y)\rangle \leq \kappa\|x-y\|^{2}, \quad \forall x, y \in C .
$$

In 1974, Deimling [3] proved the existence of fixed points of continuous $\kappa$-strongly pseudocontractive mappings in Banach spaces; see [3] for more details. We remark that the class of $\kappa$-strongly pseudocontractive mappings is independent of the class of $\kappa$-strictly pseudocontractive mappings. This can be seen from Zhou [4]. Lipschitz pseudocontractive mappings may not be $\kappa$-strictly pseudocontractive mappings, which can be seen from Chidume and Mutangadura [5].

One classical way to study nonexpansive mappings is to use contractions to approximate a nonexpansive mapping; for more details, see [6-12] and the references therein. More precisely, take $t \in(0,1)$ and define a contraction $T_{t}: C \rightarrow C$ by

$$
T_{t} x=t u+(1-t) T x, \quad \forall x \in C,
$$


where $u \in C$ is a fixed point. Banach's contraction mapping principle guarantees that $T_{t}$ has a unique fixed point $x_{t}$ in $C$. In the case of $T$ having a fixed point, Browder [7] proved that $x_{t}$ converges strongly to a fixed point of $T$ in the framework of Hilbert spaces. Reich [10] extended Browder's result to the setting of Banach spaces and proved that if $E$ is a uniformly smooth Banach space, then $x_{t}$ converges strongly to a fixed point of $T$ and the limit defines the (unique) sunny nonexpansive retraction from $C$ onto $F(T)$; see [10] for more details.

Recall that the normal Mann iterative process was introduced by Mann [13] in 1953. Recently, the construction of fixed points for nonexpansive mappings via the normal Mann iterative process has been extensively investigated by many authors. The normal Mann iterative process generates a sequence $\left\{x_{n}\right\}$ in the following manner:

$$
\left\{\begin{array}{l}
x_{1} \in C \quad \text { chosen arbitrarily, } \\
x_{n+1}=\left(1-\alpha_{n}\right) x_{n}+\alpha_{n} T x_{n}, \quad \forall n \geq 1,
\end{array}\right.
$$

where the sequence $\left\{\alpha_{n}\right\}$ is in the interval $(0,1)$.

In an infinite-dimensional Hilbert space, the normal Mann iteration algorithm has only weak convergence; see [14] for more details. In many disciplines, including economics [15], image recovery [16] and control theory [17], problems arise in infinite dimension spaces. In such problems, strong convergence is often much more desirable than weak convergence for it translates the physically tangible property so that the energy $\left\|x_{n}-x\right\|$ of the error between the iterate $x_{n}$ and the solution $x$ eventually becomes arbitrarily small.

Recently, many authors have tried to modify the normal Mann iteration process to have strong convergence for nonexpansive mappings and $\kappa$-strictly pseudocontractive mappings; see [18-36] and the references therein.

Let $D$ be a nonempty subset of $C$. Let $Q: C \rightarrow D$. $Q$ is said to be a contraction iff $Q^{2}=Q$; sunny iff for each $x \in C$ and $t \in(0,1)$, we have $Q(t x+(1-t) Q x)=Q x$; sunny nonexpansive retraction iff $Q$ sunny, nonexpansive and contraction. $K$ is said to be a nonexpansive retract of $C$ if there exists a nonexpansive retraction from $C$ onto $D$. The following result, which was established in [37] and [38], describes a characterization of sunny nonexpansive retractions on a smooth Banach space.

Let $Q: E \rightarrow C$ be a retraction, and let $j$ be the normalized duality mapping on $E$. Then the following are equivalent:

(1) $Q$ is sunny and nonexpansive;

(2) $\|Q x-Q y\|^{2} \leq\langle x-y, j(Q x-Q y)\rangle, \forall x, y \in E$;

(3) $\langle x-Q x, j(y-Q x)\rangle \leq 0, \forall x \in E, y \in C$.

In this paper, we investigate the problem of modifying the normal Mann iteration process for a family of $\kappa$-strictly pseudocontractive mappings. Strong convergence of the purposed iterative process is obtained in a real 2-uniformly Banach space. In order to prove our main results, we need the following tools.

Lemma 1.1 [1] Let E be a real 2-uniformly smooth Banach space with the best smooth constant $K$. Then the following inequality holds:

$$
\|x+y\|^{2} \leq\|x\|^{2}+2\langle y, J x\rangle+2\|K y\|^{2}, \quad \forall x, y \in E .
$$


Lemma 1.2 [31] Let C be a nonempty subset of a real 2-uniformly smooth Banach space $E$ and let $T: C \rightarrow C$ be a $\kappa$-strict pseudocontraction. For $\alpha \in(0,1)$, we define $T_{\alpha} x=(1-\alpha) x+$ $\alpha$ Tx for every $x \in C$. Then, as $\alpha \in\left(0, \frac{\kappa}{K^{2}}\right], T_{\alpha}$ is nonexpansive such that $F\left(T_{\alpha}\right)=F(T)$.

Lemma 1.3 [39] Assume that $\left\{\alpha_{n}\right\}$ is a sequence of nonnegative real numbers such that

$$
\alpha_{n+1} \leq\left(1-\gamma_{n}\right) \alpha_{n}+\delta_{n}
$$

where $\left\{\gamma_{n}\right\}$ is a sequence in $(0,1)$ and $\left\{\delta_{n}\right\}$ is a sequence such that

(i) $\sum_{n=1}^{\infty} \gamma_{n}=\infty$;

(ii) $\limsup _{n \rightarrow \infty} \delta_{n} / \gamma_{n} \leq 0$ or $\sum_{n=1}^{\infty}\left|\delta_{n}\right|<\infty$.

Then $\lim _{n \rightarrow \infty} \alpha_{n}=0$.

Lemma 1.4 [40] Let E be a real smooth Banach space. Then the following inequality holds:

$$
\|x+y\|^{2} \leq\|x\|^{2}+2\langle y, J(x+y)\rangle, \quad \forall x, y \in E .
$$

Lemma 1.5 [29] Let $E$ be a smooth Banach space and let $C$ be a nonempty convex subset of E. Given an integer $N \geq 1$, assume that $\left\{T_{i}\right\}_{i=1}^{N}: C \rightarrow C$ is a finite family of $\kappa_{i}$-strict pseudocontractions such that $\bigcap_{i=1}^{N} F\left(T_{i}\right) \neq \emptyset$. Assume that $\left\{\lambda_{i}\right\}_{i=1}^{r}$ is a positive sequence such that $\sum_{i=1}^{N} \lambda_{i}=1$. Then $F\left(\sum_{i=1}^{N} F\left(T_{i}\right)\right)=\bigcap_{i=1}^{N} F\left(T_{i}\right)$.

Lemma 1.6 [10] Let E be a real uniformly smooth Banach space and let $C$ be a nonempty closed convex subset of $E$. Let $T: C \rightarrow C$ be a nonexpansive mapping with a fixed point and let $f: C \rightarrow C$ be a contraction. For each $t \in(0,1)$, let $z_{t}$ be the unique solution of the equation $x=t f(x)+(1-t) T x$. Then $\left\{z_{t}\right\}$ converges to a fixed point of $T$ as $t \rightarrow 0$ and $Q(f)=$ $s-\lim _{t \rightarrow 0} z_{t}$ defines the unique sunny nonexpansive retraction from $C$ onto $F(T)$.

\section{Main results}

Theorem 2.1 Let $C$ be a nonempty closed convex subset of a real 2-uniformly smooth Banach space $E$ with the best smooth constant $K$ and let $N$ be some positive integer. Let $T_{i}: C \rightarrow C$ be a $\kappa_{i}$-strictly pseudocontractive mapping for each $1 \leq i \leq N$. Assume that $\bigcap_{i=1}^{N} F\left(T_{i}\right) \neq \emptyset$. Let $f$ be an $\alpha$-contractive mapping. Let $\left\{x_{n}\right\}$ be a sequence generated in the following process:

$$
\left\{\begin{array}{l}
x_{0} \in C \quad \text { arbitrarilychosen, } \\
y_{n}=\beta_{n} x_{n}+\left(1-\beta_{n}\right) \sum_{i=1}^{N} \lambda_{i} T_{i} x_{n}, \\
x_{n+1}=\alpha_{n} f\left(y_{n}\right)+\left(1-\alpha_{n}\right) y_{n}, \quad n \geq 0,
\end{array}\right.
$$

where $\left\{\alpha_{n}\right\},\left\{\beta_{n}\right\}$ and $\left\{\lambda_{i}\right\}$ are real number sequences in $[0,1]$ satisfying the following restrictions:

(a) $\sum_{n=0}^{\infty} \alpha_{n}=\infty, \lim _{n \rightarrow \infty} \alpha_{n}=0, \sum_{n=1}^{\infty}\left|\alpha_{n}-\alpha_{n-1}\right|<\infty$,

(b) $1-\frac{\kappa}{K^{2}} \leq \beta_{n} \leq \beta<1, \sum_{n=1}^{\infty}\left|\beta_{n}-\beta_{n-1}\right|<\infty$,

(c) $\sum_{n=1}^{N} \lambda_{i}=1$,

where $\beta$ is some real number, and $\kappa:=\min \left\{\kappa_{i}: 1 \leq i \leq N\right\}$. Then $\left\{x_{n}\right\}$ converges strongly as $n \rightarrow \infty$ to some point in $\bigcap_{i=1}^{N} F\left(T_{i}\right)$, which is the unique solution in $\bigcap_{i=1}^{N} F\left(T_{i}\right)$ to the 
following variational inequality:

$$
\left\langle f\left(x^{*}\right)-x^{*}, j\left(x^{*}-p\right)\right\rangle \geq 0, \quad \forall p \in \bigcap_{i=1}^{N} F\left(T_{i}\right) .
$$

Proof The proof is split into four steps.

Step 1 . Show that $\left\{x_{n}\right\}$ and $\left\{y_{n}\right\}$ are bounded.

Putting $T:=\sum_{i=1}^{N} \lambda_{i} T_{i}$, we see that $T$ is a $\kappa$-strictly pseudocontractive mapping. Indeed, we have the following:

$$
\begin{aligned}
\langle T x- & T y, j(x-y)\rangle \\
= & \lambda_{1}\left\langle T_{1} x-T_{1} y, j(x-y)\right\rangle+\lambda_{2}\left\langle T_{2} x-T_{2} y, j(x-y)\right\rangle+\cdots \\
& +\lambda_{N}\left\langle T_{N} x-T_{N} y, j(x-y)\right\rangle \\
\leq & \lambda_{1}\left(\|x-y\|^{2}-\kappa_{1}\left\|\left(I-T_{1}\right) x-\left(I-T_{1}\right) y\right\|^{2}\right) \\
& +\lambda_{2}\left(\|x-y\|^{2}-\kappa_{2}\left\|\left(I-T_{2}\right) x-\left(I-T_{2}\right) y\right\|^{2}\right)+\cdots \\
& +\lambda_{N}\left(\|x-y\|^{2}-\kappa_{N}\left\|\left(I-T_{N}\right) x-\left(I-T_{N}\right) y\right\|^{2}\right) \\
\leq & \|x-y\|^{2}-\kappa\left(\lambda_{1}\left\|\left(I-T_{1}\right) x-\left(I-T_{1}\right) y\right\|^{2}\right. \\
& \left.+\lambda_{2}\left\|\left(I-T_{2}\right) x-\left(I-T_{2}\right) y\right\|^{2}+\cdots+\lambda_{N}\left\|\left(I-T_{N}\right) x-\left(I-T_{N}\right) y\right\|^{2}\right) \\
\leq & \|x-y\|^{2}-\kappa\|(I-T) x-(I-T) y\|^{2} .
\end{aligned}
$$

This proves that $T$ is a $\kappa$-strictly pseudocontractive mapping. Fix $p \in \bigcap_{i=1}^{N} F\left(T_{i}\right)$. It follows from Lemma 1.1 that

$$
\begin{aligned}
\left\|y_{n}-p\right\|^{2}= & \left\|\left(x_{n}-p\right)+\left(1-\beta_{n}\right)\left(T x_{n}-x_{n}\right)\right\|^{2} \\
\leq & \left\|x_{n}-p\right\|^{2}+2\left(1-\beta_{n}\right)\left(T x_{n}-x_{n}, j\left(x_{n}-p\right)\right\rangle \\
& +2 K^{2}\left(1-\beta_{n}\right)^{2}\left\|\left(T x_{n}-x_{n}\right)\right\|^{2} \\
= & \left\|x_{n}-p\right\|^{2}+2\left(1-\beta_{n}\right)\left(T x_{n}-p, j\left(x_{n}-p\right)\right\rangle-2\left(1-\beta_{n}\right)\left\|x_{n}-p\right\|^{2} \\
& +2 K^{2}\left(1-\beta_{n}\right)^{2}\left\|\left(T x_{n}-x_{n}\right)\right\|^{2} \\
\leq & \left\|x_{n}-p\right\|^{2}+2\left(1-\beta_{n}\right)\left(\left\|x_{n}-p\right\|^{2}-\kappa\left\|T x_{n}-x_{n}\right\|^{2}\right) \\
& -2\left(1-\beta_{n}\right)\left\|x_{n}-p\right\|^{2}+2 K^{2}\left(1-\beta_{n}\right)^{2}\left\|\left(T x_{n}-x_{n}\right)\right\|^{2} \\
= & \left\|x_{n}-p\right\|^{2}-2\left(1-\beta_{n}\right)\left(\kappa-K^{2}\left(1-\beta_{n}\right)\right)\left\|T x_{n}-x_{n}\right\|^{2} \\
\leq & \left\|x_{n}-p\right\|^{2} .
\end{aligned}
$$

This implies that

$$
\begin{aligned}
\left\|x_{n+1}-p\right\| & =\left\|\alpha_{n}\left(f\left(y_{n}\right)-p\right)+\left(1-\alpha_{n}\right)\left(y_{n}-p\right)\right\| \\
& \leq \alpha_{n}\left\|f\left(y_{n}\right)-p\right\|+\left(1-\alpha_{n}\right)\left\|y_{n}-p\right\| \\
& \leq\left(1-\alpha_{n}(1-\alpha)\right)\left\|y_{n}-p\right\|+\alpha_{n}\|f(p)-p\|
\end{aligned}
$$




$$
\begin{aligned}
& \leq\left(1-\alpha_{n}(1-\alpha)\right)\left\|x_{n}-p\right\|+\alpha_{n}\|f(p)-p\| \\
& \leq \max \left\{\left\|x_{n}-p\right\|, \frac{\|f(p)-p\|}{1-\alpha}\right\} .
\end{aligned}
$$

This in turn implies that

$$
\left\|x_{n}-p\right\| \leq \max \left\{\left\|x_{0}-p\right\|, \frac{\|p-f(p)\|}{1-\alpha}\right\}
$$

which gives that the sequence $\left\{x_{n}\right\}$ is bounded, so is $\left\{y_{n}\right\}$. This completes step 1 .

Step 2. Show that $\left\|T_{\frac{\kappa}{K^{2}}} x_{n}-x_{n}\right\| \rightarrow 0$ as $n \rightarrow \infty$.

Put $T_{\beta_{n}} x=\beta_{n} x+\left(1-\beta_{n}\right) T x, \forall x \in C$. It follows from Lemma 1.2 that

$$
\begin{aligned}
\left\|y_{n}-y_{n-1}\right\| & =\left\|T_{\beta_{n}} x_{n}-T_{\beta_{n-1}} x_{n-1}\right\| \\
& \leq\left\|T_{\beta_{n}} x_{n}-T_{\beta_{n}} x_{n-1}\right\|+\left\|T_{\beta_{n}} x_{n-1}-T_{\beta_{n-1}} x_{n-1}\right\| \\
& \leq\left\|x_{n}-x_{n-1}\right\|+\left\|\beta_{n} x_{n-1}+\left(1-\beta_{n}\right) T x_{n-1}-\beta_{n-1} x_{n-1}-\left(1-\beta_{n-1}\right) T x_{n-1}\right\| \\
& \leq\left\|x_{n}-x_{n-1}\right\|+\left|\beta_{n}-\beta_{n-1}\right|\left\|x_{n-1}-T x_{n-1}\right\| .
\end{aligned}
$$

Notice that

$$
x_{n+1}-x_{n}=\alpha_{n}\left(f\left(y_{n}\right)-f\left(y_{n-1}\right)\right)+\left(1-\alpha_{n}\right)\left(y_{n}-y_{n-1}\right)+\left(\alpha_{n}-\alpha_{n-1}\right)\left(f\left(y_{n-1}\right)-y_{n-1}\right) .
$$

It follows from (2.2) that

$$
\begin{aligned}
& \left\|x_{n+1}-x_{n}\right\| \\
& \leq \alpha_{n}\left\|f\left(y_{n}\right)-f\left(y_{n-1}\right)\right\|+\left(1-\alpha_{n}\right)\left\|y_{n}-y_{n-1}\right\|+\left|\alpha_{n}-\alpha_{n-1}\right|\left\|f\left(y_{n-1}\right)-y_{n-1}\right\| \\
& \leq\left(1-\alpha_{n}(1-\alpha)\right)\left\|y_{n}-y_{n-1}\right\|+\left|\alpha_{n}-\alpha_{n-1}\right|\left\|f\left(y_{n-1}\right)-y_{n-1}\right\| \\
& \leq\left(1-\alpha_{n}(1-\alpha)\right)\left\|x_{n}-x_{n-1}\right\|+\left|\beta_{n}-\beta_{n-1}\right|\left\|x_{n-1}-T x_{n-1}\right\| \\
& +\left|\alpha_{n}-\alpha_{n-1}\right|\left\|f\left(y_{n-1}\right)-y_{n-1}\right\| \text {. }
\end{aligned}
$$

In view of Lemma 1.3, we obtain from the restrictions (a) and (b) that

$$
\lim _{n \rightarrow \infty}\left\|x_{n+1}-x_{n}\right\|=0
$$

Notice that

$$
x_{n+1}-x_{n}=\alpha_{n}\left(f\left(x_{n}\right)-x_{n}\right)+\left(1-\alpha_{n}\right)\left(y_{n}-x_{n}\right) .
$$

In view of the restriction (a), we obtain that $\lim _{n \rightarrow \infty}\left\|y_{n}-x_{n}\right\|=0$. On the other hand, we have $y_{n}-x_{n}=\left(1-\beta_{n}\right)\left(T x_{n}-x_{n}\right)$. This in turn implies that $\lim _{n \rightarrow \infty}\left\|T x_{n}-x_{n}\right\|=0$. It follows from the restriction (b) that

$$
\lim _{n \rightarrow \infty}\left\|T_{\frac{\kappa}{K^{2}}} x_{n}-x_{n}\right\|=0
$$

This completes step 2. 
Step 3. Show that

$$
\limsup _{n \rightarrow \infty}\left\langle z-f(z), j\left(z-x_{n}\right)\right\rangle \leq 0,
$$

where $z=Q f(z)$, where $Q$ is a sunny nonexpansive retraction from $C$ onto $\bigcap_{i=1}^{N} F\left(T_{i}\right)$, is the strong limit of the sequence $z_{t}$ defined by

$$
z_{t}=t f\left(z_{t}\right)+(1-t) T_{\frac{\kappa}{K^{2}}} z_{t}, \quad \forall t \in(0,1) .
$$

It follows that

$$
z_{t}-x_{n}=(1-t)\left(T_{\frac{\kappa}{K^{2}}} z_{t}-x_{n}\right)+t\left(f\left(z_{t}\right)-x_{n}\right), \quad \forall t \in(0,1) .
$$

For any $t \in(0,1)$, we see from Lemma 1.4 that

$$
\begin{aligned}
\left\|z_{t}-x_{n}\right\|^{2} \leq & (1-t)^{2}\left\|T_{\frac{\kappa}{K^{2}}} z_{t}-x_{n}\right\|^{2}+2 t\left\langle f\left(z_{t}\right)-x_{n}, j\left(z_{t}-x_{n}\right)\right\rangle \\
\leq & (1-t)^{2}\left(\left\|T_{\frac{\kappa}{K^{2}}} z_{t}-T_{\frac{\kappa}{K^{2}}} x_{n}\right\|^{2}+\left\|T_{\frac{\kappa}{K^{2}}} x_{n}-x_{n}\right\|^{2}\right. \\
& \left.+2\left\|T_{\frac{\kappa}{K^{2}}} z_{t}-T_{\frac{\kappa}{K^{2}}} x_{n}\right\|\left\|T_{\frac{\kappa}{K^{2}}} x_{n}-x_{n}\right\|\right)+2 t\left\langle f\left(z_{t}\right)-z_{t}, j\left(z_{t}-x_{n}\right)\right\rangle \\
& +2 t\left\langle z_{t}-x_{n}, j\left(z_{t}-x_{n}\right)\right\rangle \\
\leq & (1-t)^{2}\left\|z_{t}-x_{n}\right\|^{2}+\lambda_{n}(t)+2 t\left|f\left(z_{t}\right)-z_{t}, j\left(z_{t}-x_{n}\right)\right\rangle+2 t\left\|z_{t}-x_{n}\right\|^{2},
\end{aligned}
$$

where

$$
\lambda_{n}(t)=\left\|T_{\frac{\kappa}{K^{2}}} x_{n}-x_{n}\right\|^{2}+2\left\|z_{t}-x_{n}\right\|\left\|T_{\frac{\kappa}{K^{2}}} x_{n}-x_{n}\right\| .
$$

It follows from (2.6) that

$$
\left\langle z_{t}-f\left(z_{t}\right), j\left(z_{t}-x_{n}\right)\right\rangle \leq \frac{t}{2}\left\|z_{t}-x_{n}\right\|^{2}+\frac{1}{2 t} \lambda_{n}(t) .
$$

This implies that

$$
\limsup _{n \rightarrow \infty}\left\langle z_{t}-f\left(z_{t}\right), j\left(z_{t}-x_{n}\right)\right\rangle \leq \frac{t}{2}\left\|z_{t}-x_{n}\right\|^{2} .
$$

Since $E$ is 2-uniformly smooth, $J: E \rightarrow E^{*}$ is uniformly continuous on any bounded sets of $E$, which ensures that the $\limsup _{n \rightarrow \infty}$ and limsup $\sup _{t \rightarrow 0}$ are interchangeable, and hence

$$
\limsup _{n \rightarrow \infty}\left\langle z-f(z), j\left(z-x_{n}\right)\right\rangle \leq 0 .
$$

This shows that (2.5) holds. This completes the proof of step 3.

Step 4. Show that $x_{n} \rightarrow z$ as $n \rightarrow \infty$.

It follows from (2.1) that $\left\|y_{n}-z\right\| \leq\left\|x_{n}-z\right\|$. In view of Lemma 1.4, we see that

$$
\begin{aligned}
\left\|x_{n+1}-z\right\|^{2} & =\left\|\left(1-\alpha_{n}\right)\left(y_{n}-z\right)+\alpha_{n}\left(f\left(y_{n}\right)-z\right)\right\|^{2} \\
& \leq\left(1-\alpha_{n}\right)^{2}\left\|y_{n}-z\right\|^{2}+2 \alpha_{n}\left\langle f\left(y_{n}\right)-z, J\left(x_{n+1}-z\right)\right\rangle
\end{aligned}
$$




$$
\begin{aligned}
\leq & \left(1-\alpha_{n}\right)^{2}\left\|x_{n}-z\right\|^{2}+2 \alpha_{n}\left\langle f\left(y_{n}\right)-f(z), J\left(x_{n+1}-z\right)\right\rangle \\
& +2 \alpha_{n}\left\langle f(z)-z, J\left(x_{n+1}-z\right)\right\rangle \\
\leq & \left(\left(1-\alpha_{n}\right)^{2}+\alpha_{n} \alpha\right)\left\|x_{n}-z\right\|^{2}+\alpha_{n} \alpha\left\|x_{n+1}-z\right\|^{2}+2 \alpha_{n}\left\langle f(z)-z, J\left(x_{n+1}-z\right)\right\rangle .
\end{aligned}
$$

It then follows that

$$
\begin{aligned}
\left\|x_{n+1}-z\right\|^{2} \leq & \frac{1-(2-\alpha) \alpha_{n}+\alpha^{2}}{1-\alpha_{n} \alpha}\left\|x_{n}-z\right\|^{2}+\frac{2 \alpha_{n}}{1-\alpha_{n} \alpha}\left\langle f(z)-z, J\left(x_{n+1}-z\right)\right\rangle \\
\leq & \frac{1-(2-\alpha) \alpha_{n}}{1-\alpha_{n} \alpha}\left\|x_{n}-z\right\|^{2}+\frac{2 \alpha_{n}}{1-\alpha_{n} \alpha}\left\langle f(z)-z, J\left(x_{n+1}-z\right)\right\rangle \\
& +\frac{\alpha_{n}^{2}}{1-\alpha_{n} \alpha}\left\|x_{n}-z\right\|^{2} \\
\leq & \left(1-\frac{2(1-\alpha) \alpha_{n}}{1-\alpha_{n} \alpha}\right)\left\|x_{n}-z\right\|^{2}+\frac{2(1-\alpha) \alpha_{n}}{1-\alpha_{n} \alpha}\left(\frac{1}{1-\alpha}\left\langle f(z)-z, J\left(x_{n+1}-z\right)\right\rangle\right. \\
& \left.+\frac{\alpha_{n}}{2(1-\alpha)}\left\|x_{n}-z\right\|^{2}\right) .
\end{aligned}
$$

It follows from the restrictions (a) and (b) that

$$
\lim _{n \rightarrow \infty} \frac{2(1-\alpha) \alpha_{n}}{1-\alpha_{n} \alpha}=0, \quad \sum_{n=1}^{\infty} \frac{2(1-\alpha) \alpha_{n}}{1-\alpha_{n} \alpha}=\infty
$$

and

$$
\limsup _{n \rightarrow \infty}\left(\frac{1}{1-\alpha}\left\langle f(z)-z, J\left(x_{n+1}-z\right)\right\rangle+\frac{\alpha_{n}}{2(1-\alpha)}\left\|x_{n}-z\right\|^{2}\right) \leq 0
$$

This implies from Lemma 1.3 that $x_{n} \rightarrow z$ as $n \rightarrow \infty$. This completes the proof.

For a single mapping, we have the following.

Corollary 2.2 Let $C$ be a nonempty closed convex subset of a real 2-uniformly smooth Banach space $E$ with the best smooth constant $K$. Let $T: C \rightarrow C$ be a $\kappa$-strictly pseudocontractive mapping such that $F(T) \neq \emptyset$. Let $f$ be an $\alpha$-contractive mapping. Let $\left\{x_{n}\right\}$ be a sequence generated in the following process:

$$
\left\{\begin{array}{l}
x_{0} \in C \text { arbitrarilychosen, } \\
y_{n}=\beta_{n} x_{n}+\left(1-\beta_{n}\right) T x_{n}, \\
x_{n+1}=\alpha_{n} f\left(y_{n}\right)+\left(1-\alpha_{n}\right) y_{n}, \quad n \geq 0
\end{array}\right.
$$

where $\left\{\alpha_{n}\right\},\left\{\beta_{n}\right\}$ and $\left\{\lambda_{i}\right\}$ are real number sequences in $[0,1]$ satisfying the following restrictions:

(a) $\sum_{n=0}^{\infty} \alpha_{n}=\infty, \lim _{n \rightarrow \infty} \alpha_{n}=0, \sum_{n=1}^{\infty}\left|\alpha_{n}-\alpha_{n-1}\right|<\infty$;

(b) $1-\frac{\kappa}{K^{2}} \leq \beta_{n} \leq \beta<1, \sum_{n=1}^{\infty}\left|\beta_{n}-\beta_{n-1}\right|<\infty$,

where $\beta$ is some real number, and $\kappa:=\min \left\{\kappa_{i}: 1 \leq i \leq N\right\}$. Then $\left\{x_{n}\right\}$ converges strongly as $n \rightarrow \infty$ to some point in $F(T)$, which is the unique solution in $F(T)$, to the following 
variational inequality:

$$
\left\langle f\left(x^{*}\right)-x^{*}, j\left(x^{*}-p\right)\right\rangle \geq 0, \quad \forall p \in F(T) .
$$

If $E$ is a Hilbert space, then the best smooth constant $K=\frac{\sqrt{2}}{2}$. The following result can be deduced from Theorem 2.1 immediately.

Corollary 2.3 Let $C$ be a nonempty closed convex subset of a real Hilbert space $E$ and let $N$ be some positive integer. Let $T_{i}: C \rightarrow C$ be a $\kappa_{i}$-strictly pseudocontractive mapping for each $1 \leq i \leq N$. Assume that $\bigcap_{i=1}^{N} F\left(T_{i}\right) \neq \emptyset$. Let $f$ be an $\alpha$-contractive mapping. Let $\left\{x_{n}\right\}$ be a sequence generated in the following process:

$$
\left\{\begin{array}{l}
x_{0} \in C \quad \text { arbitrarilychosen, } \\
y_{n}=\beta_{n} x_{n}+\left(1-\beta_{n}\right) \sum_{i=1}^{N} \lambda_{i} T_{i} x_{n}, \\
x_{n+1}=\alpha_{n} f\left(y_{n}\right)+\left(1-\alpha_{n}\right) y_{n}, \quad n \geq 0,
\end{array}\right.
$$

where $\left\{\alpha_{n}\right\},\left\{\beta_{n}\right\}$ and $\left\{\lambda_{i}\right\}$ are real number sequences in $[0,1]$ satisfying the following restrictions:

(a) $\sum_{n=0}^{\infty} \alpha_{n}=\infty, \lim _{n \rightarrow \infty} \alpha_{n}=0, \sum_{n=1}^{\infty}\left|\alpha_{n}-\alpha_{n-1}\right|<\infty$;

(b) $1-2 \kappa \leq \beta_{n} \leq \beta<1, \sum_{n=1}^{\infty}\left|\beta_{n}-\beta_{n-1}\right|<\infty$;

(c) $\sum_{n=1}^{N} \lambda_{i}=1$,

where $\beta$ is some real number, and $\kappa:=\min \left\{\kappa_{i}: 1 \leq i \leq N\right\}$. Then $\left\{x_{n}\right\}$ converges strongly as $n \rightarrow \infty$ to some point in $\bigcap_{i=1}^{N} F\left(T_{i}\right)$, which is the unique solution in $\bigcap_{i=1}^{N} F\left(T_{i}\right)$, to the following variational inequality:

$$
\left\langle f\left(x^{*}\right)-x^{*}, j\left(x^{*}-p\right)\right\rangle \geq 0, \quad \forall p \in \bigcap_{i=1}^{N} F\left(T_{i}\right) .
$$

\section{Competing interests}

The authors declare that they have no competing interests.

\section{Authors' contributions}

All authors contributed equally to this manuscript. All authors read and approved the final manuscript.

\section{Author details}

${ }^{1}$ Department of Mathematics, Hangzhou Normal University, Hangzhou, 310036, China. ²Department of Mathematics, Gyeongsang National University, Jinju, 660-701, Korea. ${ }^{3}$ Department of Mathematics, School of Science, Beijing Jiaotong University, Beijing, 100044, China. ${ }^{4}$ Department of Mathematics, Shijiazhuang University, Shijiazhuang, 050035, China.

\section{Acknowledgements}

This research was supported by the Natural Science Foundation of Hebei Province (A2010001943), the Science Foundation of Shijiazhuang Science and Technology Bureau (121130971) and the Science Foundation of Beijing Jiaotong University (2011YJS075). The authors are grateful to the referees for their valuable comments and suggestions which improved the contents of the article.

\section{Received: 13 February 2013 Accepted: 19 April 2013 Published: 2 May 2013}

\section{References}

1. Cioranescu, I: Geometry of Banach Spaces, Duality Mappings and Nonlinear Problems. Kluwer Academic, Dordrecht (1990)

2. Browder, FE, Petryshyn, WV: Construction of fixed points of nonlinear mappings in Hilbert spaces. J. Math. Anal. Appl. 20, 197-228 (1967)

3. Deimling, K: Zeros of accretive operators. Manuscr. Math. 13, 365-374 (1974) 
4. Zhou, $\mathrm{H}$ : Convergence theorems of fixed points for Lipschitz pseudo-contractions in Hilbert spaces. J. Math. Anal. Appl. 343, 546-556 (2008)

5. Chidume, CE, Mutangadura, SA: An example on the Mann iteration method for Lipschitz pseodo-contractions. Proc Am. Math. Soc. 129, 2359-2363 (2001)

6. Qin, X, Su, Y: Approximation of a zero point of accretive operator in Banach spaces. J. Math. Anal. Appl. 329, 415-424 (2007)

7. Browder, FE: Convergence of approximants to fixed points of nonexpansive nonlinear mappings in Banach space. Arch. Ration. Mech. Anal. 24, 82-90 (1967)

8. Qin, X, Cho, YJ, Kang, SM: Approximating zeros of monotone operators by proximal point algorithm. J. Glob. Optim. $46,75-87(2000)$

9. Moudafi, A: Viscosity approximation methods for fixed-points problems. J. Math. Anal. Appl. 241, 46-55 (2000)

10. Reich, S: Strong convergence theorems for resolvents of accretive operators in Banach spaces. J. Math. Anal. Appl. 75 , 287-292 (1980)

11. Marino, G, et al.: Strong convergence of the modified Mann iterative method for strict pseudo-contractions. Comput. Math. Appl. 57, 455-465 (2009)

12. Cho, SY, Kang, SM: Approximation of common solutions of variational inequalities via strict pseudocontractions. Acta Math. Sci. 32, 1607-1618 (2012)

13. Mann, WR: Mean value methods in iteration. Proc. Am. Math. Soc. 4, 506-510 (1953)

14. Genel, A, Lindenstruss, J: An example concerning fixed points. Isr. J. Math. 22, 81-86 (1975)

15. Khan, MA, Yannelis, NC: Equilibrium Theory in Infinite Dimensional Spaces. Springer, New York (1991)

16. Combettes, PL: The convex feasibility problem in image recovery. In: Hawkes, P (ed.) Advanced in Imaging and Electron Physcis, vol. 95, pp. 155-270. Academic Press, New York (1996)

17. Fattorini, HO: Infinite-Dimensional Optimization and Control Theory. Cambridge University Press, Cambridge (1999)

18. Qin, X, Cho, YJ, Kang, SM, Shang, MJ: A hybrid iterative scheme for asymptotically $\kappa$-strict pseudo-contractions in Hilbert spaces. Nonlinear Anal. 70, 1902-1911 (2009)

19. Jung, JS: Strong convergence of iterative methods for k-strictly pseudo-contractive mappings in Hilbert spaces. Appl. Math. Comput. 215, 3746-3753 (2010)

20. Cho, SY: Approximation of fixed points of pseudocontraction semigroups based on a viscosity iterative process. Appl. Math. Lett. 24, 224-228 (2011)

21. Cho, SY: Hybrid projection algorithms for treating common fixed points of a family of demicontinuous pseudocontractions. Appl. Math. Lett. 25, 854-857 (2012)

22. Luo, $\mathrm{H}$, Wang, Y: Iterative approximation for the common solutions of a infinite variational inequality system for inverse-strongly accretive mappings. J. Math. Comput. Sci. 2, 1660-1670 (2012)

23. Ye, J, Huang, J: Strong convergence theorems for fixed point problems and generalized equilibrium problems of three relatively quasi-nonexpansive mappings in Banach spaces. J. Math. Comput. Sci. 1, 1-18 (2011)

24. Qin, X, Cho, YJ, Kang, SM: Convergence theorems of common elements for equilibrium problems and fixed point problems in Banach spaces. J. Comput. Appl. Math. 225, 20-30 (2009)

25. $\mathrm{LV}, \mathrm{S}, \mathrm{Wu}, \mathrm{C}$ : Convergence of iterative algorithms for a generalized variational inequality and a nonexpansive mapping. Eng. Math. Lett. 1, 44-57 (2012)

26. Kang, SM, Cho, SY, Liu, Z: Convergence of iterative sequences for generalized equilibrium problems involving inverse-strongly monotone mappings. J. Inequal. Appl. 2010, Article ID 827082 (2010)

27. Zegeye, $\mathrm{H}$, Shahzad, N: Strong convergence theorem for a common point of solution of variational inequality and fixed point problem. Adv. Fixed Point Theory 2, 374-397 (2012)

28. Qin, X, Cho, YJ, Kang, SM: Viscosity approximation methods for generalized equilibrium problems and fixed point problems with applications. Nonlinear Anal. 72, 99-112 (2010)

29. Zhou, $\mathrm{H}$ : Convergence theorems of common fixed points for a finite family of Lipschitz pseudocontractions in Banach spaces. Nonlinear Anal. 68, 2977-2983 (2008)

30. Zhou, $\mathrm{H}$ : Strong convergence of an explicit iterative algorithm for continuous pseudo-contractions in Banach spaces. Nonlinear Anal. 70, 4039-4046 (2009)

31. Zhou, H: Convergence theorems for $\lambda$-strict pseudo-contractions in 2-uniformly smooth Banach spaces. Nonlinear Anal. 69, 3160-3173 (2008)

32. Qin, $X$, Chang, SS, Cho, YJ: Iterative methods for generalized equilibrium problems and fixed point problems with applications. Nonlinear Anal. 11, 2963-2972 (2010)

33. Qin, X, Cho, YJ, Kang, SM, Zhou, HY: Convergence of a modified Halpern-type iteration algorithm for quasi- $\phi$-nonexpansive mappings. Appl. Math. Lett. 22, 1051-1055 (2009)

34. Bunyawat, A, Suantai, S: Strong convergence theorems for variational inequalities and fixed points of a countable family of nonexpansive mappings. Fixed Point Theory Appl. 2011, Article ID 47 (2011)

35. Hao, $Y$, Wang, $X$, Tong, A: Weak and strong convergence theorems for two finite families of asymptotically nonexpansive mappings in Banach spaces. Adv. Fixed Point Theory 2, 417-432 (2012)

36. Qin, X, Shang, M, Su, Y: Strong convergence of a general iterative algorithm for equilibrium problems and variational inequality problems. Math. Comput. Model. 48, 1033-1046 (2008)

37. Bruck, RE: Nonexpansive projections on subsets of Banach spaces. Pac. J. Math. 47, $341-355$ (1973)

38. Reich, S: Asymptotic behavior of contractions in Banach spaces. J. Math. Anal. Appl. 44, 57-70 (1973)

39. Liu, LS: Ishikawa and Mann iteration process with errors for nonlinear strongly accretive mappings in Banach spaces. J. Math. Anal. Appl. 194, 114-125 (1995)

40. Jung, JS, Cho, YJ, Zhou, H: Iterative methods with mixed errors for perturbed $m$-accretive operator equations in arbitrary Banach spaces. Math. Comput. Model. 35, 55-62 (2002)

doi:10.1186/1687-1812-2013-117

Cite this article as: Qing et al.: Strong convergence of an iterative process for a family of strictly pseudocontractive mappings. Fixed Point Theory and Applications 2013 2013:117. 